\section{Prognoseabschätzung bei CLL}

Zur Prognoseabschätzung bei chronischer lymphatischer Leukämie (CLL) reicht das klinische Stagingsystem nicht mehr aus - Testung und Anwendung neuer molekularbiologischer Therapien erfordern präzisere Analysen.

— in internationales Konsortium entwi- ckelte einen verbindlichen prognostischen Index für die CLL (CLL-IPI) mit den wichtigsten prognostischen Parametern. In einer systematischen Datenrecherche wurden Studien zur CLL identifiziert, die die definierten Kriterien erfüllten. Endpunkt war das Gesamtüberleben

(OS). Insgesamt gingen Daten von 3.472 therapienaiven Patienten ein, 2.308 in ein Trainings- und 1.164 in ein ValidierungsSet. Zudem dienten die Daten von $838 \mathrm{~Pa}$ tienten der Mayo Clinic (Rochester, MN/ USA) und 416 Patienten einer skandinavischen bevölkerungsbasierten Fall-Kontroll-Studie der externen Validierung.

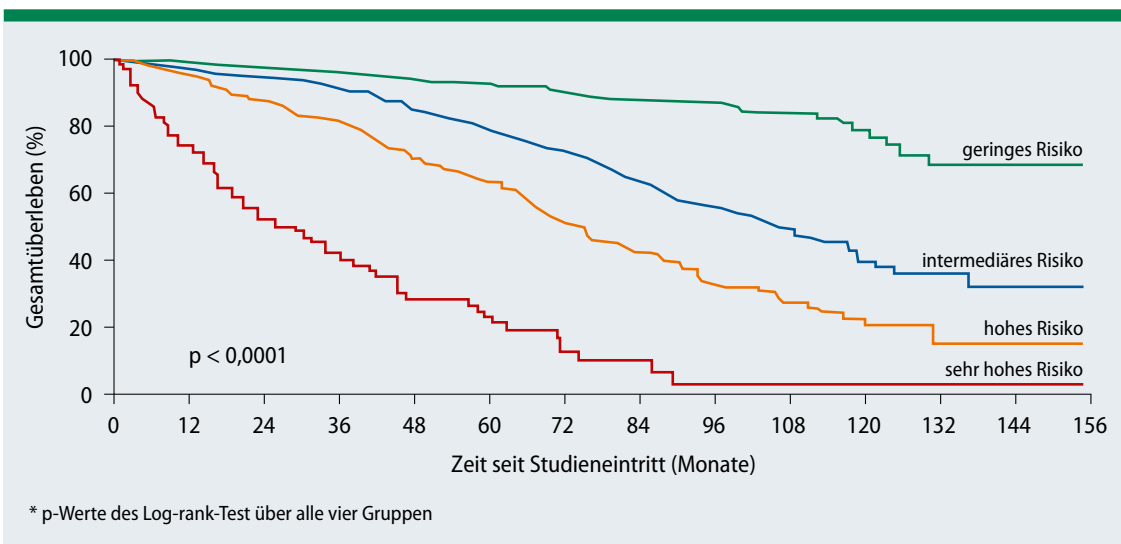

Abb. 1: Der CLL-IPI differenziert deutlich Überlebensunterschiede abhängig vom Risiko.

\section{CLL: Bendamustin-Rituximab weniger toxisch?}

Die Standard-Chemoimmuntherapie mit Fludarabin, Cyclophosphamid und Rituximab (FCR) bei fortgeschrittener CLL eignet sich nur für fitte Patienten. Ist BR als weniger toxische Kombination eine Alternative?

$\mathrm{M}$ it der Einführung der Chemoimmuntherapie hat sich die Situation für jüngere Patienten mit chronischer lymphatischer Leukämie (CLL) erheblich verbessert. Bei jüngeren Patienten in gutem Allgemeinzustand ist die Frontlinetherapie mit FCR mittlerweile Standard. Doch sie ist mit erheblichen Nebenwirkungen verbunden, etwa schwerer Hämatotoxizität oder schweren Infektionen.

In einer randomisierten Phase-IIIStudie mit Nichtunterlegenheitsdesign wurde deshalb die Kombination von Bendamustin plus Rituximab (BR) mit FCR verglichen. 561 therapienaive CLL-
Patienten (33-81 Jahre) ohne 17p-Deletion erhielten 6 Zyklen FCR $(\mathrm{n}=282)$ oder BR ( $\mathrm{n}=279)$. Primärer Endpunkt war das progressionsfreie Überleben (PFS) mit dem Ziel, die Nichtunterlegenheit von BR gegenüber der Standardtherapie zu zeigen.

Nach median 37,1 Monaten war das mediane PFS unter FCR länger als unter BR (55,2 vs. 41,7 Monate; Hazard Ratio 1,643; 90,4\%-Konfidenzintervall [KI] $1,308-2,064)$. Weil die obere Grenze des 90,4\%-KI höher als der vorgegebene Wert von 1,388 war, konnte die Nichtunterlegenheit nicht bestätigt werden.
Im Traingsset fanden sich 5 unabhängige prognostische Faktoren: TP53-Status (keine Anomalien vs. del17p und/oder TP53-Mutation), IGHV-Mutationsstatus (mutiert vs. nicht mutiert), Serum- $\beta 2$ Mikroglobulin ( $\leq 3,5 \mathrm{mg} / \mathrm{L}$ vs. $>3,5 \mathrm{mg} / \mathrm{L})$, klinisches Stadium (Binet A oder Rai 0 vs. Binet B-C oder Rai I-IV) und Alter ( $\leq$ vs. $>65$ Jahre).

Aus den gewichteten Faktoren wurde ein prognostischer Index errechnet, der im Trainingsset 4 Risikogruppen anzeigte, mit signifikant unterschiedlichen 5-Jahres-OS-Raten: niedriges Risiko (93,2\%), intermediäres Risiko (79,3\%), hohes Risiko (63,3\%), sehr hohes Risiko (23,3\%; Log-rank-Test über alle 4 Risikogruppen $\mathrm{p}<0,0001$; C-Statistik $\mathrm{c}=0,723$ ) (Abb. 1).

In der internen und externen Validierung wurden die Risikogruppen bestätigt.

Fazit: Der CLL-IPI integriert genetische, biochemische und klinische Parameter in ein prognostisches Modell. 4 prognostische Subgruppen können unterschieden werden.

Kathrin von Kieseritzky

International CLL-IPI working group. An international prognostic index for patients with chronic lymphocytic leukaemia (CLL-IPI): a metaanalysis of individual patient data. Lancet Oncol. 2016;17(6):779-90.

Unter FCR wurden häufiger schwere Neutropenie und Infektionen beobachtet als unter BR ( 84 vs. $59 \%$ sowie 39 vs. $25 \%)$. Häufigere Infektionen unter FCR betrafen verstärkt ältere Patienten ( $>65$ Jahre).

Fazit: Obwohl - wahrscheinlich aufgrund der geringeren Toxizität - mehr Patienten unter BR alle 6 Therapiezyklen durchliefen als unter FCR, konnte eine Nichtunterlegenheit nicht gezeigt werden. FCR bleibt der Standard in der Ersttherapie bei CLL-Patienten in guter körperlicher Verfassung. Kathrin von Kieseritzky

Eichhorst B et al. First-line chemoimmunotherapy with bendamustine and rituximab versus fludarabine, cyclophosphamide, and rituximab in patients with advanced chronic lymphocytic leukaemia (CLL10): an international, open-label, randomised, phase 3, non-inferiority trial. Lancet Oncol. 2016;17(7):928-42. 